\section{Lyapunov Theory for 2-D Nonlinear Roesser Models: Application to Asymptotic and Exponential Stability}

\author{
Nima Yeganefar, Nader Yeganefar, Mariem Ghamgui, and \\ Emmanuel Moulay
}

\begin{abstract}
This technical note deals with a general class of discrete 2-D possibly nonlinear systems based on the Roesser model. We first motivate the introduction of Lyapunov type definitions of asymptotic and exponential stability. This will allow us to introduce and discuss several particularities that cannot be found in 1-D systems. Once this background has been carefully designed, we develop different Lyapunov theorems in order to check asymptotic and exponential stability of nonlinear 2-D systems. Finally we propose the first converse Lyapunov theorem in the case of exponential stability.
\end{abstract}

Index Terms - Converse Lyapunov theorem, Lyapunov functions, $n \mathrm{D}$ systems, nonlinear discrete systems.

\section{INTRODUCTION}

\section{A. Historical Context}

The first studies on multidimensional systems started in the mid 1970s. The focus was mainly on linear systems and transfer functions. Instead of working with a transfer function, which depends on one independent variable, they looked at polynomial functions of 2 and more generally $n$ variables. The notion of stability has also been investigated in terms of BIBO stability (bounded input bounded output) [1]. This led to the famous stability criterion which roughly states that the transfer function is BIBO stable for systems devoid of poles outside the stability region. It was quoted as a necessary and sufficient condition, led to several stability tests [2], [3], and broadened to include exponential stability in [4]. However Goodman later showed in [5] that the condition is necessary only if the transfer function does not present a particular type of point called nonessential singularity of the second kind. It is worth noting that these points have no equivalence in the 1-D case.

The first state-space models were introduced a bit later with the work of Roesser [6] and Fornasini-Marchesini [7], [8], who also proposed a definition of asymptotic stability relevant to their model. With the introduction of state space models and the development of several techniques based on linear matrix inequalities (LMIs) and Lyapunov techniques, there was a growing interest in 2-D systems ([9]-[15], etc.).

\section{B. Stability Background for 1-D Systems}

Consider the following dynamical system: $x(i+1)=f(x(i))$, where $i \in \mathbb{Z}, x$ is a sequence with values in some $\mathbb{R}^{n}, f: \mathbb{R}^{n} \rightarrow \mathbb{R}^{n}$ is a map such that $f(0)=0$, and the initial condition is given by $x(0)=x_{0}$. Recall the well-known stability definitions ([16]):

Manuscript received November 23, 2011; revised May 23, 2012; accepted September 18, 2012. Date of publication September 20, 2012; date of current version April 18, 2013. Recommended by Associate Editor H. L. Trentelman.

$\mathrm{Ni}$. Yeganefar and M. Ghamgui are with the University of Poitiers, LIAS, Poitiers 86022, France. (e-mail: nima.yeganefar@univ-poitiers.fr; mariem.ghamgui@etu.univ-poitiers.fr).

Na. Yeganefar is with the University of Provence, CMI (UMR 6632), Technopole Chateau-Gombert, Marseille 13453, France (e-mail: nader. yeganefar@cmi.univ-mrs.fr).

M. Ghamgui is with the University of Poitiers, LIAS, ENSIP-LAII, Poitiers 86022, France (e-mail: mariem.ghamgui@etu.univ-poitiers.fr).

E. Moulay is with the University of Poitiers, Xlim (UMR-CNRS 6172), Futuroscope Chasseneuil 86962, France (e-mail: emmanuel.moulay@univpoitiers.fr).

Digital Object Identifier 10.1109/TAC.2012.2220012 (i) $x_{e}$ is called stable in the sense of Lyapunov if for all $\epsilon>0$, there exists $\delta=\delta(\epsilon)>0$ such that if $\|x(0)\|<\delta$, then for all $i \geq 0$, $\|x(i)\|<\epsilon$.

(ii) $x_{e}$ is called attractive if for any trajectory $x$, we have $x(i) \rightarrow 0$ as $i \rightarrow+\infty$.

(iii) $x_{e}$ is called asymptotically stable if it is stable and attractive.

(iv) $x_{e}$ is called exponentially stable if there exist constants $q \in$ $(0,1)$ and $C>0$ such that for every trajectory $x$ and all $i \geq 0$, we have $\|x(i)\| \leq C q^{i}\|x(0)\|$.

Obviously, exponential stability implies asymptotic stability, and asymptotic stability implies attractivity (by definition). The converse implications are generally not true [17, Section 40], but they hold for linear systems.

The main goal of this technical note is to consider similar definitions for multidimensional systems and develop a corresponding Lyapunov theory.

\section{Initial Conditions for 2-D Systems}

Let us first introduce the studied system, a generalization of the model introduced by Roesser [6]:

$$
\left[\begin{array}{l}
x^{h}(i+1, j) \\
x^{v}(i, j+1)
\end{array}\right]=f\left(x^{h}(i, j), x^{v}(i, j)\right)
$$

where $f: \mathbb{R}^{n} \times \mathbb{R}^{m} \rightarrow \mathbb{R}^{n} \times \mathbb{R}^{m}$ is a given function with $f(0,0)=$ $(0,0), x=\left(x^{h}, x^{v}\right) \in \mathbb{R}^{n} \times \mathbb{R}^{m}$ and $(i, j) \in \mathbb{Z}^{2}$. To have unique solutions, we have to specify a subset $I \subset \mathbb{Z}^{2}$ on which initial conditions have to be given, but contrary to the 1-D case, there is no obvious choice. The first possible choice is to notice that in the 1-D case, there is a natural notion of past (the set of negative integers), present (at 0 ), and future (the set of positive integers). This is the approach adopted by Fornasini-Marchesini ([7], [8]) for 2-D systems: these authors consider initial conditions given on the subset $I=\left\{(i, j) \in \mathbb{Z}^{2}, i+j=0\right\}$, and, the "future" being the subset $\left\{(i, j) \in \mathbb{Z}^{2}, i+j>0\right\}$, they study the behavior of trajectories as $i+j \rightarrow+\infty$. More specifically, the system is called asymptotically stable if given any bounded initial condition on $I$, the corresponding trajectory goes to zero as $i+j \rightarrow+\infty$. Note that in this definition, one has to consider only bounded initial conditions, otherwise one may have unbounded trajectories. Several technical notes have since extended the stability definitions and discussed the importance of the initial conditions. In the more general case, [18] studied the stability for proper cones, and recently [19] extended the concept to the notion of time-relevant stability, where one of the dimensions (usually time) plays a specific role for stability.

The second possible choice for initial conditions, which also appears in the literature ([4], [14], [20], [21]), is to give $x^{h}$ and $x^{v}$ on the positive semi-axes (i.e., $x^{h}(0, j)$, and $x^{v}(i, 0)$ for $i, j \in \mathbb{N}$ ), and to study the behavior of trajectories in the first quadrant $\mathbb{N}^{2}$ as $i+j \rightarrow+\infty$. Our work will take place in this framework. We would like to mention here a key difference between this approach and the Fornasini-Marchesini approach, which we feel has not been highlighted in previous works. If the initial conditions are given on the positive semi-axes, and if we want the trajectories to have some convergence property as $i+j \rightarrow+\infty$, then the initial conditions have to satisfy at least this property, too. For example, one cannot hope to require attractivity (i.e., $x(i, j) \rightarrow 0$ as $i+j \rightarrow+\infty)$ for any bounded initial conditions, because attractivity implies in particular that $x^{h}(0, j) \rightarrow 0$ as $j \rightarrow+\infty$ and $x^{v}(i, 0) \rightarrow 0$ as $i \rightarrow+\infty$.

\section{Contribution}

Let us now explain what is really new in this technical note, and why we need to revisit these notions of stability which have already 
long been studied. Firstly, as recalled in the beginning of this introduction, in the 1-D case, asymptotic stability encapsulates two properties, namely stability in the sense of Lyapunov and attractivity. However, in the 2-D case, most authors usually forget about the stability part (with the exception of [20]), and only use attractivity. Secondly, there is no satisfying definition of exponential stability in the 2-D case (see Section II for more on this). Thirdly, works on the stability of 2-D systems deal almost exclusively with linear systems. This could also explain the first two points above, because for 2-D linear systems, attractivity should imply exponential stability and stability in the sense of Lyapunov; a proof of this is however missing in the literature.

Our aim here is to study general nonlinear systems such as (1), with initial conditions given on the positive semi-axes. For these systems, we will define a notion of asymptotic stability which generalizes the existing notion for 1-D systems (see Section II). We will also define a notion of exponential stability. This definition is completely new and shows an interesting feature concerning 2-D systems which had not yet been documented (see Proposition 2.5). In Section III, we will develop theoremsà la Lyapunov that are in coherence with our proposed definitions. These theorems are tools to assess the different types of stability introduced. Moreover, we will introduce the first converse Lyapunov theorem in the exponential case for 2-D discrete nonlinear systems (for the 1-D case, one can refer to [22]).

\section{Notations}

Let us briefly examine the notations that will be used in this technical note. A continuous function $\alpha: \mathbb{R}^{+} \rightarrow \mathbb{R}^{+}$is said to be of class $\mathcal{K}$, if $\alpha(0)=0$ and $\alpha$ is strictly increasing. $\alpha$ is said to be of class $\mathcal{K}_{\infty}$ if it is of class $\mathcal{K}$ and if $\lim _{t \rightarrow \infty} \alpha(t)=\infty$.

For any $q \in] 0,1\left[\right.$, let us denote by $E_{q}\left(\mathbb{N}, \mathbb{R}^{n}\right)$ the set of $\mathbb{R}^{n}$-valued sequences which decay exponentially at rate $q$, that is, a sequence $u=(u(k))_{k \in \mathbb{N}}$ belongs to $E_{q}\left(\mathbb{N}, \mathbb{R}^{n}\right)$ if for some constant $C$, we have $\|u(k)\| \leq C q^{k}, \quad \forall k$. We endow $E_{q}\left(\mathbb{N}, \mathbb{R}^{n}\right)$ with two different norms. First, the sup norm $\|u\|_{\infty}=\sup _{k}\|u(k)\|$ and second $\|u\|_{q}=\sup _{k}\left(q^{-k}\|u(k)\|\right)$. Note that $\|u\|_{\infty} \leq\|u\|_{q}$ but the two norms are not equivalent.

\section{STABILITY Definitions}

Recall that we want to define stability properties for the following nonlinear 2-D Roesser model

$$
\left[\begin{array}{l}
x^{h}(i+1, j) \\
x^{v}(i, j+1)
\end{array}\right]=f\left(x^{h}(i, j), x^{v}(i, j)\right)
$$

where $f: \mathbb{R}^{n} \times \mathbb{R}^{m} \rightarrow \mathbb{R}^{n} \times \mathbb{R}^{m}$ is a given function with $f(0,0)=$ $(0,0), x=\left(x^{h}, x^{v}\right) \in \mathbb{R}^{n} \times \mathbb{R}^{m},(i, j) \in \mathbb{N}^{2}$, and with initial conditions the sequences $x^{h}(0, \cdot), x^{v}(\cdot, 0)$.

Definition $2.1\left(\epsilon-\delta\right.$ Stability): The point $x_{e}=0$ is said to be stable (in the sense of Lyapunov) if for all $\epsilon>0$, there exists $\delta(\epsilon)>0 \mathrm{such}$ that if $\|x(0, j)\|<\delta,\|x(i, 0)\|<\delta$ then $\|x(i, j)\|<\epsilon$ for all $i, j>0$.

Definition 2.2 (Asymptotic Stability): The point $x_{e}=0$ is said to be asymptotically stable (in the sense of Lyapunov) if:

1) $x_{e}=0$ is stable (in the sense of Definition 2.1);

2) $\lim _{i+j \rightarrow \infty} x(i, j)=0$ when $\lim _{j \rightarrow \infty} x^{h}(0, j)=0$ and $\lim _{i \rightarrow \infty} x^{v}(i, 0)=0$. This property will be called attractivity as in the 1-D case.

Remark 1: In order to verify asymptotic stability, one needs to first check the stability condition (where the initial conditions need not go to 0 at infinity) and then to check that $\lim _{i+j \rightarrow \infty} x(i, j)=0$ when $\lim _{j \rightarrow \infty} x^{h}(0, j)=0$ and $\lim _{i \rightarrow \infty} x^{v}(i, 0)=0$. This is a fundamental difference with the 1-D case where asymptotic stability implies that all trajectories go to 0 at infinity whatever the initial conditions are.
In the 2-D case, taking the initial conditions in the first quadrant, one cannot hope to have every trajectory approaching 0 simply because it may not be the case for the initial conditions.

Remark 2: Usually in the literature, the notion of stability is set aside to retain only the attractivity condition in Definition 2.2, and without mentioning that the initial conditions have to go to 0 at infinity.

Let us now introduce the definition of exponential stability. One has to keep in mind that it must be an extension of the 1-D case and at least imply asymptotic stability as defined above.

Definition 2.3 (Exponential Stability): The equilibrium point $x_{e}=$ 0 of system (2) is said to be exponentially stable if there are constants $q \in(0,1)$ and $M>0$ such that for any initial conditions $x^{h}(0, \cdot)$ and $x^{v}(\cdot, 0)$, and for all $(i, j) \in \mathbb{N}^{2}$, we have

$$
\|x(i, j)\| \leq M\left(\sum_{r=0}^{i} \frac{\left\|x^{v}(r, 0)\right\|}{q^{r+1}}+\sum_{s=0}^{j} \frac{\left\|x^{h}(0, s)\right\|}{q^{s+1}}\right) q^{i+j} .
$$

Some comments on this definition are in order. First, it is easy to verify that each term $x(i, j)$ is determined by the knowledge of $x^{v}(r, 0)$ and $x^{h}(0, s)$, for $r=0, \ldots i$, and $s=0, \ldots, j$, so that it is natural to express the bound of $x(i, j)$ in terms of these quantities. Next, we have the following property, as in the 1-D case:

Proposition 2.4: Exponential stability implies asymptotic stability (Definition 2.2).

Proof: Stability comes from the fact that for a given $\epsilon>0$, there exists a $\delta=\epsilon(1-q) / 4 M>0$ such that if for all $i, j>0$, $\left\|x^{h}(0, j)\right\|<\delta$ and $\left\|x^{v}(i, 0)\right\|<\delta$, then

$$
\begin{aligned}
\|x(i, j)\| & \leq M\left(\sum_{k=0}^{j} \frac{\left\|x^{h}(0, k)\right\|}{q^{k+1}}+\sum_{k=0}^{i} \frac{\left\|x^{v}(k, 0)\right\|}{q^{k+1}}\right) q^{i+j} \\
& \leq M \delta\left(\sum_{k=0}^{j} \frac{1}{q^{k+1}}+\sum_{k=0}^{i} \frac{1}{q^{k+1}}\right) q^{i+j} \\
& \leq M \delta\left(\frac{q^{j-1}+q^{i-1}-2 q^{i+j}}{1-q}\right) \leq \epsilon .
\end{aligned}
$$

Attractivity follows by similar arguments.

To further motivate our definition, consider a special example of system (2) where $n=m=1$ and $f$ is given by an upper triangular matrix with a single eigenvalue $q$

$$
\left[\begin{array}{l}
x^{h}(i+1, j) \\
x^{v}(i, j+1)
\end{array}\right]=\left[\begin{array}{ll}
q & 1 \\
0 & q
\end{array}\right]\left[\begin{array}{l}
x^{h}(i, j) \\
x^{v}(i, j)
\end{array}\right] .
$$

If $q \in(0,1)$, then 0 is an exponentially stable equilibrium point as expected. Indeed, with initial conditions still on the positive semi-axes, we can explicitly compute the solutions: $x^{h}(i, j)=$ $q^{i} x^{h}(0, j)+q^{i+j} \sum_{k=0}^{i-1} x^{v}(k, 0) / q^{k+1}$ and $x^{v}(i, j)=q^{j} x^{v}(i, 0)$. Exponential stability follows easily.

We would also like to point out a striking feature of 2-D systems which does not occur in the 1-D case: in the general exponentially stable case of Definition 2.3, even if the initial conditions decay exponentially at rate $q$, the trajectory itself does not necessarily decay at rate $q$, but only at rate $q^{\prime}$, for any $q^{\prime}>q$. We record this fact in the following proposition:

Proposition 2.5: Assume that the equilibrium point 0 is exponentially stable for system (2) in the sense of Definition 2.3. Assume also that $x$ is a trajectory of system (2), whose initial conditions decay exponentially at rate $q$. Then for any $q^{\prime}>q, x$ decays exponentially at rate $q^{\prime}$, but $x$ does not necessarily decay at rate $q$.

Proof: As the initial conditions decay exponentially at rate $q$, we can find some positive constant $C$ such that for all $r, s \in \mathbb{N}$, we have 
$\left\|x^{v}(r, 0)\right\| \leq C q^{r}$ and $\left\|x^{h}(0, s)\right\| \leq C q^{s}$. Now, by exponential stability, we have for all $i, j \in \mathbb{N}$

$$
\begin{aligned}
\|x(i, j)\| & \leq M\left(\sum_{r=0}^{i} \frac{\left\|x^{v}(r, 0)\right\|}{q^{r+1}}+\sum_{s=0}^{j} \frac{\left\|x^{h}(0, s)\right\|}{q^{s+1}}\right) q^{i+j} \\
& \leq M C\left(\sum_{r=0}^{i} \frac{q^{r}}{q^{r+1}}+\sum_{s=0}^{j} \frac{q^{s}}{q^{s+1}}\right) q^{i+j} \\
& =M C(i+j+2) q^{i+j-1} \leq C^{\prime}\left(q^{\prime}\right)^{i+j}
\end{aligned}
$$

for some positive constant $C^{\prime}>0$ (which depends on $q$ and $q^{\prime}$ ). This means precisely that $x$ decays exponentially at rate $q^{\prime}$. To see that $x$ does not necessarily decay at rate $q$, consider again the upper triangular linear case (3). Take for example $x^{v}(i, 0)=q^{i}$ and $x^{h}(0, j)=q^{j}$ for $i, j \in \mathbb{N}$. Then $x^{h}(i, j)=q^{i+j}+i q^{i+j-1}$, which shows that $x^{h}$ does not decay at rate $q$.

To conclude this section, let us briefly comment on the existing definitions of exponential stability for 2-D systems. One of the only works dealing with this problem is given by Pandolfi in [4]. Later in [23], the authors extended this definition to repetitive systems but we are not discussing this special type of multidimensional systems in this technical note. It is interesting to point out that Pandolfi takes the initial conditions on the positive semi-axes and proposes the following definition: the equilibrium point $x_{e}=0$ is exponentially stable if there exists a constant $M>0$ such that for some $q \in(0,1)$, all trajectories $x(i, j)$ satisfy:

$$
\|x(i, j)\| \leq M\left(q^{i} \max _{0<j^{\prime}<j}\left\|x^{h}\left(0, j^{\prime}\right)\right\|+q^{j} \max _{0<i^{\prime}<i}\left\|x^{v}\left(i^{\prime}, 0\right)\right\|\right) .
$$

With this definition, however, it is not guaranteed that $\lim _{i+j \rightarrow \infty} x(i, j)=0$ but only $\lim _{(i \text { and } j) \rightarrow \infty} x(i, j)=0$. Moreover, the trivial solution of system (3) is not exponentially stable in this sense.

One could also try the bound $\|x(i, j)\| \leq M q^{i+j}$ where $M$ is a constant which depends on the initial conditions. This is what has been recently proposed in the continuous case [24]. It is however not sufficient for us, because with this definition, it is not guaranteed that exponential stability will imply asymptotic stability in the way we want to define it. The attractivity condition is easily verified but not the $\epsilon-\delta$ stability condition. For this last condition, the bound of the initial conditions needs to be explicitly taken into account in the definition of exponential stability. Moreover, the linear upper triangular case (3) does not satisfy this condition.

\section{MAIN RESULTS}

\section{A. Direct Theorems}

The first theorem introduced in this section has been applied in the literature but a complete proof is missing if one chooses the above definition. The proof proposed here shares the same philosophy that was first, as far as we know, introduced in [9], that is, showing that the energy stored along the lines $\left\{(i, j) \in \mathbb{N}^{2} \mid i+j=r\right\}$ of an asymptotically stable system is decreasing. This idea was later extended by Liu in [20] for a system with some nonlinearities and applied to systems with delays in [13]. Here we provide a different proof, easier to follow and in coherence with our definition. Note also that in all the subsequent theorems of this section the assumption that the initial conditions have finite support is required.
Theorem 3.1: Let $V^{h}, V^{v}$ be continuous functions from $\mathbb{R}^{n} \rightarrow \mathbb{R}$, $\mathbb{R}^{m} \rightarrow \mathbb{R}$, respectively, such that for all $\left(x^{h}, x^{v}\right) \in \mathbb{R}^{n} \times \mathbb{R}^{m}$ :

$$
\begin{gathered}
\alpha_{h}\left(\left\|x^{h}\right\|\right) \leq V^{h}\left(x^{h}\right) \leq \beta_{h}\left(\left\|x^{h}\right\|\right) \\
\alpha_{v}\left(\left\|x^{v}\right\|\right) \leq V^{v}\left(x^{v}\right) \leq \beta_{v}\left(\left\|x^{v}\right\|\right)
\end{gathered}
$$

where $\alpha_{h}, \alpha_{v}, \beta_{h}, \beta_{v}$ are functions of class $\mathcal{K}$. Define $V\left(x^{h}, x^{v}\right)=$ $V^{h}\left(x^{h}\right)+V^{v}\left(x^{v}\right)$ and $\Delta V$ as the increment of $V$ along the trajectories of (2) by

$$
\begin{aligned}
\Delta V \triangleq V^{h}\left(x^{h}(i+1, j)\right) & -V^{h}\left(x^{h}(i, j)\right) \\
& +V^{v}\left(x^{v}(i, j+1)\right)-V^{v}\left(\left(x^{v}(i, j)\right) .\right.
\end{aligned}
$$

If $\Delta V \leq-\gamma(\|x\|)$, where $\gamma$ is a function of class $\mathcal{K}$ then the equilibrium $x_{e}=0$ of system (2), with the initial conditions taken on finite support, is asymptotically stable (Definition 2.2).

Remark 3: Conditions (4) also implies that there exist class $\mathcal{K}$ functions $\alpha, \beta$, such that $\alpha(\|x\|) \leq V(x) \leq \beta(\|x\|)$.

Proof: Suppose that $\Delta V<-\gamma(\|x\|)$ and (4) holds, by hypothesis, we can write

$$
\begin{aligned}
& V^{h}\left(x^{h}(i+1, j)\right)+V^{v}\left(x^{v}(i, j+1)\right) \\
& \leq V^{h}\left(x^{h}(i, j)\right)+V^{v}\left(x^{v}(i, j)\right) .
\end{aligned}
$$

Let $E(r)=\sum_{(i+j=r)} V\left(x^{h}(i, j), x^{v}(i, j)\right)$ denotes the 'energy' stored on the line $\left\{(i, j) \in \mathbb{N}^{2} \mid i+j=r\right\}$. It follows from (6) and the positivity of $\gamma$ that

$$
E(r) \geq E(r+1)-V^{h}\left(x^{h}(0, r+1)\right)-V^{v}\left(x^{v}(r+1,0)\right) .
$$

Now remember that our initial conditions are defined in a finite set. Let us call $L>0$ the highest bound of this set $\left(\right.$ i.e., $x^{h}(0, j)=x^{v}(i, 0)=$ 0 for all $i, j>L$ ) and consider the case when $r=i+j>L$. This implies that $E(r)-E(r+1) \geq 0$ which means that the energy $E(r)$ is decreasing for all $r>L$. If $r<L, E(r+1)-E(r) \leq$ $V^{h}\left(x^{h}(0, r+1)\right)+V^{v}\left(x^{v}(r+1,0)\right)$. By definition and using the positive definiteness of $V, E(0)=0$. Adding the last inequalities for $r<L$ gives us

$$
E(r) \leq \sum_{i=1}^{r} V^{h}\left(x^{h}(0, i)\right)+V^{v}\left(x^{v}(i, 0)\right)
$$

$V^{h}$ and $V^{v}$ are continuous functions with $V^{h}(0)=V^{v}(0)=0$, so by continuity, $V^{h}\left(x^{h}(0, i)\right)$ and $V^{v}\left(x^{v}(0, i)\right)$ can be as small as desired considering small initial conditions $x^{h}(0, i)$ and $x^{v}(i, 0)$. It means that for all $\epsilon>0$ there exists a $\delta$ such that if $\left\|x^{h}(0, j)\right\|<\delta$ and $\left\|x^{v}(i, 0)\right\|<\delta$

$$
\max _{r<I}(E(r)) \leq \sum_{i=1}^{r} V^{h}\left(x^{h}(0, i)\right)+V^{v}\left(x^{v}(i, 0)\right) \leq \epsilon .
$$

For $r>L$ as $E(r)$ is decreasing, $\max _{r \geq 0} E(r) \leq \epsilon$ stands, therefore

$$
\begin{aligned}
\alpha\left(\left\|\left(x^{h}(i, j), x^{v}(i, j)\right)\right\|\right) & \leq V\left(x^{h}(i, j), x^{v}(i, j)\right) \\
& \leq \max _{r \geq 0} E(r) \leq \epsilon .
\end{aligned}
$$

We conclude from Definition 2.2 that the system (2) is stable.

To conclude the proof we need to show that $\lim _{i+j \rightarrow \infty}\|x(i, j)\|=$ 0 . For $r>L, E(r)$ is a decreasing positive series that converges to a given limit, hence $\lim _{r \rightarrow \infty}(E(r)-E(r+1))=0$. But going back to inequality (6), observe that for $r>L$

$$
E(r)-E(r+1) \geq(r+1) \gamma(\|x(i, j)\|) \geq \gamma(\|x(i, j)\|) \geq 0 .
$$


This proves that $\lim _{i+j \rightarrow \infty} \gamma(\|x(i, j)\|)=0$, hence as $\gamma$ is of class $\mathcal{K}$, it leads to $\lim _{i+j \rightarrow \infty}\|x(i, j)\|=0$ which concludes the proof.

Theorem 3.2: Let $V^{h}, V^{v}$ be continuous functions from $\mathbb{R}^{n} \rightarrow \mathbb{R}$, $\mathbb{R}^{m} \rightarrow \mathbb{R}$, respectively, such that for all $\left(x^{h}, x^{v}\right) \in \mathbb{R}^{n} \times \mathbb{R}^{m}$

$$
\begin{aligned}
& a\left\|x^{h}\right\|^{p} \leq V^{h}\left(x^{h}\right) \leq b\left\|x^{h}\right\|^{p} \\
& a\left\|x^{v}\right\|^{p} \leq V^{v}\left(x^{v}\right) \leq b\left\|x^{v}\right\|^{p}
\end{aligned}
$$

where $a, b$ and $p$ are positive constants. Define $V\left(x^{h}, x^{v}\right)=V^{h}\left(x^{h}\right)+$ $V^{v}\left(x^{v}\right)$. If there exists a constant $0<\alpha<1$ such that for any trajectory $(x(i, j))$ solution of system (2) with initial conditions taken on finite support $\Delta V \leq-\alpha V$ where $\Delta V$ is defined in (5) then, $x_{e}=0$ is exponentially stable.

Proof: For simplicity, we will only consider $p=1$ but the general case is similar. Let $x(i, j)$ be a solution of system (2) with initial conditions $x^{h}(0, \cdot)$ and $x^{v}(\cdot, 0)$ with finite support. Consider the quantity $E(r)=\sum_{i+j=r} V(x(i, j))$ (or $\sum_{i+j=r} V_{h}^{1 / p}\left(x^{h}\right)+V_{v}^{1 / p}\left(x^{v}\right)$ in the general case) which represents the energy stored on the line $\left\{(i, j) \in \mathbb{N}^{2} \mid i+j=r\right\}$ and let $q=1-\alpha$. With a similar argument than the previous proof, one can show that

$$
\begin{aligned}
E(r+1)-E(r) \leq- & \alpha E(r) \\
& +V^{h}\left(x^{h}(0, r+1)\right)+V^{v}\left(x^{v}(r+1,0)\right) .
\end{aligned}
$$

By induction on $r$, it follows that:

$$
\begin{aligned}
E(r) & \leq q^{r} E(0)+\sum_{j=1}^{r} q^{r-j} V^{h}\left(x^{h}(0, j)\right)+\sum_{i=1}^{r} q^{r-i} V^{v}\left(x^{v}(i, 0)\right) \\
& =\sum_{j=0}^{r} q^{r-j} V^{h}\left(x^{h}(0, j)\right)+\sum_{i=0}^{r} q^{r-i} V^{v}\left(x^{v}(i, 0)\right) .
\end{aligned}
$$

The use of inequality (7) leads to

$$
E(r) \leq b \sum_{j=0}^{r} q^{r-j}\left\|x^{h}(0, j)\right\|+b \sum_{i=0}^{r} q^{r-i}\left\|x^{v}(i, 0)\right\| .
$$

As the initial conditions are with finite support, there is an $L$ such that for $r \geq L$, the initial conditions are equally 0 . Therefore for all $r \geq 0$

$$
E(r) \leq q^{r}\left(b \sum_{j=0}^{L} q^{-j}\left\|x^{h}(0, j)\right\|+b \sum_{i=0}^{L} q^{-i}\left\|x^{v}(i, 0)\right\|\right) .
$$

We may rewrite this as

$$
E(r) \leq M^{\prime}\left(\sum_{k=0}^{L} \frac{\left\|x^{h}(0, k)\right\|}{q^{k+1}}+\sum_{k=0}^{L} \frac{\left\|x^{v}(k, 0)\right\|}{q^{k+1}}\right) q^{r}
$$

where $M^{\prime}=b q$ is a constant. To conclude the proof, let us use the first part of inequality (7) which gives

$$
\begin{aligned}
\|x(i, j)\| & \leq a^{-1} V\left(x(i, j) \leq a^{-1} E(i+j)\right. \\
& \leq M\left(\sum_{k=0}^{L} \frac{\left\|x^{h}(0, k)\right\|}{q^{k+1}}+\sum_{k=0}^{L} \frac{\left\|x^{v}(k, 0)\right\|}{q^{k+1}}\right) q^{i+j} .
\end{aligned}
$$

This is exactly the bound given in Definition 2.3 as the initial conditions are taken with the finite support $[0, L]$ and $M=a^{-1} M^{\prime}$.

\section{B. Example}

Let us illustrate our first theorems in a nonlinear case with a simple example. Consider the system

$$
\left\{\begin{array}{l}
x^{h}(i+1, j)=\rho\left(\left\|x^{h}(i, j)\right\|\right) x^{h}(i, j)+\frac{1}{8} x^{v}(i, j) \\
x^{v}(i, j+1)=\rho\left(\left\|x^{v}(i, j)\right\|\right) x^{v}(i, j)
\end{array}\right.
$$

where $\rho:[0, \infty[\rightarrow[0, \infty[$ is a nonlinear function such that $\rho(0)=0$ and $|\rho| \leq 1 / 2$. Take the Lyapunov function candidate $V\left(x^{h}, x^{v}\right)=$ $\left\|x^{h}\right\|^{2}+\left\|x^{v}\right\|^{2}=V^{h}\left(x^{h}\right)+V^{v}\left(x^{v}\right) \cdot V$ obviously verifies the first part of Theorem 3.2. Computing the quantity $\Delta V$ (see (5)) gives

$$
\begin{aligned}
\Delta V=\left\|\rho\left(\left\|x^{h}(i, j)\right\|\right) x^{h}(i, j)+\frac{1}{8} x^{v}(i, j)\right\|^{2} \\
\quad-\left\|x^{h}(i, j)\right\|^{2}+\left\|\rho\left(\left\|x^{v}(i, j)\right\|\right) x^{v}(i, j)\right\|^{2}-\left\|x^{v}(i, j)\right\|^{2} .
\end{aligned}
$$

Using the facts that

$$
\begin{aligned}
& \left\|\rho\left(\left\|x^{h}(i, j)\right\|\right) x^{h}(i, j)+\frac{1}{8} x^{v}(i, j)\right\|^{2} \\
& \leq\left(\left\|\rho\left(\left\|x^{h}(i, j)\right\|\right) x^{h}(i, j)\right\|+\left\|\frac{1}{8} x^{v}(i, j)\right\|\right)^{2} \\
& \leq 2\left(\left\|\rho\left(\left\|x^{h}(i, j)\right\|\right) x^{h}(i, j)\right\|^{2}+\left\|\frac{1}{8} x^{v}(i, j)\right\|^{2}\right)
\end{aligned}
$$

and that $|\rho| \leq 1 / 2$, one easily gets $\Delta V \leq-(1 / 2) V$. By Theorem 3.2, the system is exponentially stable (if the initial conditions have a finite support).

\section{Converse Theorem}

The next theorem is the first converse Lyapunov theorem introduced for 2-D systems. We assume that system (2) is exponentially stable. Roughly speaking, we have to find a function $V$ (or rather a "functional" here) such that its variation along the trajectories of our system is bounded by the values of $V$ along these trajectories. To define these notions properly, we begin with some general considerations.

We can view $\left(\mathbb{R}^{n}\right)^{\mathbb{N}} \times\left(\mathbb{R}^{m}\right)^{\mathbb{N}}$ as the space of initial conditions of system (2): if $\left(u^{h}, u^{v}\right) \in\left(\mathbb{R}^{n}\right)^{\mathbb{N}} \times\left(\mathbb{R}^{m}\right)^{\mathbb{N}}$, then there is a unique trajectory $x$ of system (2) such that $x^{h}(0, \cdot)=u^{h}$ and $x^{v}(\cdot, 0)=u^{v}$. Conversely, if $x=\left(x^{h}, x^{v}\right)$ is a trajectory of system (2), then for all $(i, j) \in \mathbb{N}^{2}, x$ defines an element $\left(u^{h}, u^{v}\right)$ in $\left(\mathbb{R}^{n}\right)^{\mathbb{N}} \times\left(\mathbb{R}^{m}\right)^{\mathbb{N}}$, namely $u^{h}=x^{h}(i, \cdot+j)$ and $u^{v}=x^{v}(\cdot+i, j)$. Note that for fixed $(i, j)$, the trajectory corresponding to these initial conditions, to be denoted by $x_{i j}$, is simply given by $x_{i j}(\cdot, \cdot)=x(\cdot+i, \cdot+j)$. Let $E$ be the subset of $\left(\mathbb{R}^{n}\right)^{\mathbb{N}} \times\left(\mathbb{R}^{m}\right)^{\mathbb{N}}$ which contains all initial conditions $\left(u^{h}, u^{v}\right)$ such that if $x$ is the corresponding trajectory, then there exists a number $q^{\prime} \in(0,1)$ such that for all $(i, j), x_{i j}$ decays at rate $q^{\prime}$. Let us emphasize that, by definition, any trajectory starting at $E$ is in particular exponentially decaying at some rate $q^{\prime}$. By our discussion in Section II (see Proposition 2.5), we know that $E$ contains $E_{q}\left(\mathbb{N}, \mathbb{R}^{n}\right) \times E_{q}\left(\mathbb{N}, \mathbb{R}^{m}\right)$.

Now, let $V: E \rightarrow \mathbb{R}$ be a function defined on this subspace of initial conditions. If $x$ is an exponentially decaying trajectory of system (2), we define $V$ along $x$ to be the (double) sequence $(i, j) \mapsto V\left(x^{h}(i, \cdot+\right.$ $\left.j), x^{v}(\cdot+i, j)\right)$. We would also like to define the variation $\Delta V$ of $V$ along $x$. To this end, we assume that $V$ can be written in the following form: $V=V^{h}+V^{v}$, where $V^{h}, V^{v}: E \rightarrow \mathbb{R}$ are functions such that if $\left(u^{h}, u^{v}\right) \in E$ and $x$ is the corresponding trajectory, then $V^{h}\left(u^{h}, u^{v}\right)$ (respectively $V^{v}\left(u^{h}, u^{v}\right)$ ) depends only on $x^{h}$ (respectively on $x^{v}$ ). For such a function $V$, its variation $\Delta V$ along a trajectory $x$ is the double sequence defined for all $(i, j)$ by

$$
\begin{aligned}
& \Delta V=V^{h}\left(x^{h}(i+1, \cdot+j), x^{v}(\cdot+i+1, j)\right) \\
& +V^{v}\left(x^{h}(i, \cdot+j+1), x^{v}(\cdot+i, j+1)\right)-V\left(x^{h}(i, \cdot+j), x^{v}(\cdot+i, j)\right)
\end{aligned}
$$

which is the same as

$$
\begin{aligned}
\Delta V= & V^{h}\left(x^{h}(i+1, \cdot+j), x^{v}(\cdot+i+1, j)\right) \\
& -V^{h}\left(x^{h}(i, \cdot+j), x^{v}(\cdot+i, j)\right) \\
& +V^{v}\left(x^{h}(i, \cdot+j+1), x^{v}(\cdot+i, j+1)\right) \\
& -V^{v}\left(x^{h}(i, \cdot+j), x^{v}(\cdot+i, j)\right) .
\end{aligned}
$$


With these preliminaries in mind, we can state our result.

Theorem 3.3: Assume that system (2) is exponentially stable. Then there exists a function $V: E \rightarrow \mathbb{R}$ having the following properties:

1) There are two positive constants $C_{1}$ and $C_{2}$ such that for all $\left(u^{h}, u^{v}\right) \in E_{q}\left(\mathbb{N}, \mathbb{R}^{n}\right) \times E_{q}\left(\mathbb{N}, \mathbb{R}^{m}\right)$, we have

$C_{1}\left(\left\|u^{h}\right\|_{\infty}+\left\|u^{v}\right\|_{\infty}\right) \leq V\left(u^{h}, u^{v}\right) \leq C_{2}\left(\left\|u^{h}\right\|_{q}+\left\|u^{v}\right\|_{q}\right)$

2) There are two functions $V^{h}, V^{v}: E \rightarrow \mathbb{R}$ such that $V=$ $V^{h}+V^{v}$ and for any $\left(u^{h}, u^{v}\right) \in E$ with corresponding trajectory $x, V^{h}\left(u^{h}, u^{v}\right)$ (respectively $V^{v}\left(u^{h}, u^{v}\right)$ ) depends only on $x^{h}$ (respectively on $x^{v}$ ).

3) There is a positive constant $\alpha$ such that along any exponentially decaying trajectory of system (2) starting at $E$, we have $\Delta V \leq$ $-\alpha V$.

Proof: For $\left(u^{h}, u^{v}\right) \in E$, we let $x$ be the corresponding trajectory; $x$ is exponentially decaying at some rate $q^{\prime} \in(0,1)$. We define $V^{h}$ by $V^{h}\left(u^{h}, u^{v}\right)=\sum_{i, j=0}^{\infty}\left\|x^{h}(i, j)\right\|$. As $x$ decays exponentially, $V^{h}$ is well defined. We can also define $V^{v}$ in a similar way, and setting $V=V^{h}+V^{v}$, we see that $V$ satisfies the second property of our theorem.

The first property of the theorem is also easily verified with the given norms. First $V=V^{h}+V^{v}$ verifies the first part of the inequality with $C_{1}=1$. To see why, observe that

$$
\sum_{i, j=0}^{\infty}\left\|x^{h}(i, j)\right\| \geq \sum_{i=0}^{\infty}\left\|u^{h}(i)\right\| \geq\left\|u^{h}\right\|_{\infty} .
$$

The second part of inequality comes from first observing that for any sequence $u,\|u(k)\| \leq\|u\|_{q} q^{k}$. Now, by exponential stability (see Definition 2.3), we know that we can find some constant $M>0$ such that

$$
\begin{aligned}
\|x(i, j)\| & \leq M q^{i+j}\left(\sum_{r=0}^{i} \frac{\left\|x^{v}(r, 0)\right\|}{q^{r+1}}+\sum_{s=0}^{j} \frac{\left\|x^{h}(0, s)\right\|}{q^{s+1}}\right) \\
& =M q^{i+j}\left(\sum_{r=0}^{i} \frac{\left\|u^{v}(r)\right\|}{q^{r+1}}+\sum_{s=0}^{j} \frac{\left\|u^{h}(s)\right\|}{q^{s+1}}\right) \\
& \leq M q^{i+j-1}\left((i+1)\left\|u^{v}\right\|_{q}+(j+1)\left\|u^{h}\right\|_{q}\right)
\end{aligned}
$$

which leads to: $V\left(x^{h}, x^{v}\right) \leq M \sum_{i, j=0}^{\infty}(i+1) q^{i+j}\left(\left\|u^{h}\right\|_{q}+\left\|u^{v}\right\|_{q}\right)$, so that $V$ verifies the desired inequality.

To prove the last property, we have to compute, for fixed $(i, j)$, terms such as $V^{h}\left(x^{h}\left(i, \cdot+j, x^{v}(\cdot+i, j)\right)\right.$ for a trajectory $x$ of system (2). To achieve this, we just notice that the trajectory $\tilde{x}=x_{i j}$ corresponding to the initial conditions $\left(x^{h}(i, \cdot+j), x^{v}(\cdot+i, j)\right)$ is given by $\tilde{x}(\cdot, \cdot)=$ $x(\cdot+i, \cdot+j)$. This implies that $V^{h}\left(x^{h}(i \cdot \cdot+j), x^{v}(\cdot+i, j)\right)=$ $\sum_{k, l}\left\|x^{h}(k+i, l+j)\right\|$, and similar expressions can be found to compute the other terms involved in $\Delta V$ and $V$ along the trajectory $x$. Thus, we have

$$
\begin{aligned}
\Delta V= & \sum_{k, l=0}^{\infty}\left[\left\|x^{h}(k+i+1, l+j)\right\|-\left\|x^{h}(k+i, l+j)\right\|\right] \\
& +\sum_{k, l=0}^{\infty}\left[\left\|x^{v}(k+i, l+j+1)\right\|-\left\|x^{v}(k+i, l+j)\right\|\right] \\
= & -\sum_{l=0}^{\infty}\left\|x^{h}(i, l+j)\right\|-\sum_{k=0}^{\infty}\left\|x^{h}(k+i, j)\right\| .
\end{aligned}
$$

It follows that we have to find some $\alpha>0$ satisfying:

$$
\begin{aligned}
\alpha\left(\sum_{k, l=0}^{\infty} \| x^{h}(k+i, l+j)\right. & \left.+\sum_{k, l=0}^{\infty}\left\|x^{v}(k+i, l+j)\right\|\right) \\
\leq & \sum_{l=0}^{\infty}\left\|x^{h}(i, l+j)\right\|+\sum_{k=0}^{\infty}\left\|x^{h}(k+i, j)\right\| .
\end{aligned}
$$

Now, the exponential stability condition of Definition 2.3 applied to $\tilde{x}$ yields

$$
\begin{aligned}
\| x^{h}(k+i, l & +j) \| \\
& \leq M q^{k+l}\left[\sum_{s=0}^{l} \frac{\left\|x^{h}(i, s+j)\right\|}{q^{s+1}}+\sum_{r=0}^{k} \frac{\left\|x^{v}(r+i, j)\right\|}{q^{r+1}}\right] .
\end{aligned}
$$

Summing over $k$ and $l$, we get

$$
\begin{aligned}
\sum_{k, l=0}^{\infty}\left\|x^{h}(k+i, l+j)\right\| \leq & M \sum_{k, l=0}^{\infty} q^{k+l} \sum_{s=0}^{l} \frac{\left\|x^{h}(i, s+j)\right\|}{q^{s+1}} \\
& +M \sum_{k, l=0}^{\infty} q^{k+l} \sum_{r=0}^{k} \frac{\left\|x^{v}(r+i, j)\right\|}{q^{r+1}}
\end{aligned}
$$

The first term in the right hand side of this inequality may be dealt with as

$$
\begin{aligned}
& \sum_{k, l=0}^{\infty} q^{k+l} \sum_{s=0}^{l} \frac{\left\|x^{h}(i, s+j)\right\|}{q^{s+1}} \\
& \quad=\left(\sum_{k=0}^{\infty} q^{k}\right) \sum_{l=0}^{\infty} \sum_{s=0}^{l} \frac{\left\|x^{h}(i, s+j)\right\|}{q^{s+1}} \\
& =\frac{1}{q-1} \sum_{s=0}^{\infty} \sum_{l=s}^{\infty} q^{l} \frac{\left\|x^{h}(i, s+j)\right\|}{q^{s+1}} \\
& =\frac{1}{q-1} \sum_{s=0}^{\infty} \frac{\left\|x^{h}(i, s+j)\right\|}{q^{s+1}} \sum_{l=s}^{\infty} q^{l} \\
& =\frac{1}{q(q-1)^{2}} \sum_{s=0}^{\infty}\left\|x^{h}(i, s+j)\right\| .
\end{aligned}
$$

The second term in the right hand side of inequality (9) can be computed in the same way. Finally, we can argue in a similar way to estimate $\sum_{k, l=0}^{\infty}\left\|x^{v}(k+i, l+j)\right\|$, and conclude that $V$ satisfies the third property of our theorem with $\alpha=q(q-1)^{2} / 2 M$.

\section{CONCLUSION}

Let us highlight the contributions of this technical note. Definitions of stability, asymptotic stability and exponential stability have been provided and the coherences/differences with the ones in the 1-D case have been discussed. In the second part of the technical note we provided two different Lyapunov theorems which give sufficient conditions to check the proposed definitions. Finally the first converse theorem has been provided in the exponential case. This opens new possibilities for multidimensional systems and solid basis in order to extend some well-known techniques from the 1-D to the 2-D case.

\section{REFERENCES}

[1] J. Shanks, S. Treitel, and J. Justice, "Stability and synthesis of twodimensional recursive filters," IEEE Trans. Audio Electroacoust., vol. AU-20, no. 2, pp. 115-128, 1972. 
[2] T. Huang, "Stability of two-dimensional recursive filters," IEEE Trans. Audio Electroacoust., vol. AU-20, no. 2, pp. 158-163, 1972.

[3] B. Anderson and E. Jury, "Stability test for two-dimensional recursive filters," IEEE Trans. Audio Electroacoust., vol. AU-21, no. 4, pp. 366-372, 1973.

[4] L. Pandolfi, "Exponential stability of 2-D systems," Syst. Control Lett., vol. 4, pp. 381-385, 1984.

[5] D. Goodman, "Some stability properties of two dimensional linear shift-invariant digital filters," IEEE Trans. Circuits Syst., vol. CAS-24, no. 4, pp. 201-208, Apr. 1977.

[6] R. Roesser, "Discrete state-space model for linear image processing," IEEE Trans. Autom. Control, vol. 20, no. 1, pp. 1-10, 1975.

[7] E. Fornasini and G. Marchesini, "State-space realization theory of twodimensional filters," IEEE Trans. Autom. Control, vol. 21, no. 4, pp. 484-492, 1976.

[8] E. Fornasini and G. Marchesini, "Doubly-indexed dynamical systems: State-space models and structural properties," Math. Syst. Theory, vol. 12 , pp. 59-72, 1978.

[9] T. Hinamoto, "The Fornasini-Marchesini model with no over flow oscillations and its application to 2-D digital filter design," in Proc. IEEE Int. Symp. Circuits Syst. (ISCAS), Portland, OR, 1989, vol. 3, pp. $1680-1683$.

[10] C. Du and L. Xie, "Stability analysis and stabilization of uncertain two-dimensional discrete systems: An LMI approach," IEEE Trans. Circuits Syst. I, vol. 46, no. 11, pp. 1371-1374, Nov. 1999.

[11] P.-A. Bliman, "Lyapunov equation for the stability of 2-D systems," Multidimensional Syst. Signal Process., vol. 13, no. 2, pp. 201-222, 2002.

[12] K. Galkowski, J. Lam, S. Xu, and Z. Lin, "LMI approach to state-feedback stabilization of multidimensional systems," Int. J. Control, vol. 76, no. 14, pp. 1428-1436, 2003

[13] W. Paszke, J. Lam, K. Galkowski, S. Xu, and Z. Lin, "Robust stability and stabilization of 2-D discrete state-delayed systems," Syst. Control Lett., vol. 51, pp. 277-291, 2004.

[14] T. Kaczorek, "LMI approach to stability of 2-D positive systems," Multidimensional Syst. Signal Process., vol. 20, no. 1, pp. 39-54, 2009.

[15] D. N. Avelli, P. Rapisarda, and P. Rocha, "Lyapunov stability of 2-D finite-dimensional behaviours," Int. J. Control, vol. 84, no. 4, pp. 737-745, 2011.

[16] H. Khalil, Nonlinear Systems, 3rd ed. Englewood Cliffs, NJ: Prentice-Hall, 2002.

[17] W. Hahn, Stability of Motion. Berlin - New York: Springer Verlag, 1967.

[18] M. Valcher, "Characteristic cones and stability properties of two-dimensional autonomous behaviors," IEEE Trans. Circuits Syst. I, vol. 47 , no. 3, pp. 290-302, Mar. 2000.

[19] D. Napp, P. Rapisarda, and P. Rocha, "Time-relevant stability of 2-D systems," Autom., vol. 47, no. 11, pp. 2373-2382, 2011.

[20] D. Liu and A. N. Michel, "Stability analysis of state-space realizations for two-dimensional filters with overflow nonlinearities," IEEE Trans. Circuits Syst. I, vol. 41, pp. 127-137, 1994.

[21] J. Bochniak and K. Galkowski, "LMI-based analysis for continuousdiscrete linear shift invariant nD-systems," J. Circuits, Syst. Comput., vol. 14, no. 2, pp. 307-332, 2005.

[22] Z.-P. Jiang and Y. Wang, "A converse Lyapunov theorem for discretetime systems with disturbances," Syst. Control Lett., vol. 45, pp. 49-58, 2002.

[23] M. Dymkov, I. Gaishun, K. Galkowski, E. Rogers, and D. Owens, "Exponential stability of discrete linear repetitive processes," Int. J. Control, vol. 75, no. 12, pp. 861-869, 2002.

[24] M. Ghamgui, N. Yeganefar, O. Bachelier, and D. Mehdi, "Exponential stability conditions for 2-D continuous state-delayed systems," in Proc. nDS '11, 7th Int. Wkshp Multidimensional Syst., 2011, pp. 1-5.

\section{Affine Characterizations of Minimal and Mode-Dependent Dwell-Times for Uncertain Linear Switched Systems}

\author{
Corentin Briat and Alexandre Seuret
}

\begin{abstract}
An alternative approach for minimum and mode-dependent dwell-time characterization for switched systems is derived. While minimum-dwell time results require the subsystems to be asymptotically stable, mode-dependent dwell-time results can consider unstable subsystems and dwell-times within a, possibly unbounded, range of values. The proposed approach is related to Lyapunov looped-functionals, a new type of functionals leading to stability conditions affine in the system matrices, unlike standard results for minimum dwell-time. These conditions are expressed as infinite-dimensional LMIs which can be solved using recent polynomial optimization techniques such as sum-of-squares. The specific structure of the conditions is finally utilized in order to derive dwell-time stability results for uncertain switched systems. Several examples illustrate the efficiency of the approach.
\end{abstract}

Index Terms-Dwell-time, looped-functionals, sum of squares, switched systems, uncertain systems.

\section{INTRODUCTION}

Switched systems [1]-[9] are an important subclass of hybrid systems for which the system dynamics are selected among a countable family of subsystems. They are very powerful modeling tools for several real world processes, like congestion modeling and control in networks [10]-[13], switching control laws [4], electromechanical systems [14], networked control systems [15], electrical devices/circuits [16], [17], etc. These systems exhibit interesting behaviors motivating their analysis: for instance, switching between asymptotically stable subsystems does not always result in an overall stable system [18], [19]. Conversely, switching between unstable subsystems may result in asymptotically stable trajectories [18], [19]. It is well-known that in the case of asymptotically stable linear subsystems, when their matrices commute or can be expressed in an upper triangular form via a common similarity transformation, stability of the overall switched system under arbitrary switching is actually equivalent to the existence of a quadratic common Lyapunov function [4], [19]. In all the other cases, the existence of a quadratic common Lyapunov function is only sufficient, albeit the existence of a common (not necessarily quadratic) Lyapunov function is necessary. When no common Lyapunov function exists, approaches based for instance on polyhedral Lyapunov functions [20], [21] or switched Lyapunov functions [3], may be considered instead. The main difficulty arising from the use of switched Lyapunov functions lies in the discontinuities of the Lyapunov function level at

Manuscript received June 12, 2012; revised June 12, 2012 and September 06, 2012; accepted September 07, 2012. Date of publication September 20, 2012; date of current version April 18, 2013. This work was supported by the FeedNetBack project, FP7-ICT-2007-2. Recommended by Associate Editor P. Shi.

C. Briat is with the Department of Biosystems Science and Engineering (D-BSSE), Swiss Federal Institute of Technology-Zürich (ETH-Z), Basel 4058, Switzerland (e-mail: corentin@briat.info; briatc@bsse.ethz.ch; url: http://www.briat.info).

A. Seuret is with the Department of Automatic Control, Gipsa-lab, Grenoble BP 46, 38402, France (e-mail: alexandre.seuret@gipsa-lab.grenoble-inp.fr).

Color versions of one or more of the figures in this technical note are available online at $\mathrm{http}: / /$ ieeexplore.ieee.org.

Digital Object Identifier 10.1109/TAC.2012.2220031 